\title{
Burkhard Dirks (ed.): Die Notfallmedizin
}

\author{
Springer, 2007, ISBN: 978-3-540-25608-3
}

\author{
Norbert Kleinsasser
}

Received: 10 December 2008 / Accepted: 10 December 2008 / Published online: 31 January 2009

(C) Springer-Verlag 2009

The book "Die Notfallmedizin" [Emergency Medicine] was edited by Burkhard Dirks and published by the Springer company in 2007. It is written in the German language and contains 588 pages with 359 partially coloured illustrations.

The author points out his aim to create a book that includes all principles of emergency medicine. Consequently, it addresses physicians specialised in emergency medicine and members of almost all medical specialities as well. The book is structured according to the guidelines of the German National Medical Association and the curriculum for the education of medical students in emergency medicine.

Four main chapters cover basic knowledge, frequent emergencies arranged by symptoms and medical specialities, respectively, as well as organisational principals of emergency medicine in Germany. Within the first three chapters, the otorhinolaryngologist may gather information about how to treat emergencies in his/her field and examine the perfect approach in cases of emergency medicine in detail.

In Chap. 1, basic medical knowledge is depicted. For the ENT specialist the article on disorders of vital functions is particularly interesting to quickly brush up relevant physiological and pathophysiological knowledge. How to secure the respiratory tract is described also. Here, the correct application of oxygen, the clearing of the upper respiratory tract, and the techniques of intubation are summarised. This is followed by adequate resuscitation techniques.

Chapter 2 is arranged by symptoms and their therapies: unconsciousness, respiratory disorders, acute thoracic pain, shock and traumatic injuries.

In the third chapter, most of the symptoms of the previous chapter may be found again, but now they are aligned according to medical specialities. Additional articles cover special fields of medicine, e.g., specific emergencies of the head and neck. Nasal bleeding, fractures and obstruction of the airways are described in seven pages, which may appear to be not detailed enough as seen from our point of view. Furthermore, a special section of otological, nasal and laryngological emergencies is missing completely. It might have given assistance to all physicians in this special part of emergency medicine.

The fourth chapter clarifies the complicated structure of the German emergency medical system, for those interested.

In summary, the book may be an excellent guidebook for physicians working in the field of emergency medicine. For the otorhinolaryngologist, general aspects of emergency medicine are presented in vast details, but specific issues of the head and neck are neglected.

N. Kleinsasser ( $\square)$

Department of Otolaryngology, Head and Neck Surgery,

University of Wuerzburg, Josef-Schneider-Str. 11,

Haus B2, 97080 Würzburg, Germany

e-mail: Kleinsasser_N@klinik.uni-wuerzburg.de 